JOURNAL OF THEORETICAL

AND APPLIED MECHANICS

57, 3, pp. 765-777, Warsaw 2019

DOI: $10.15632 /$ jtam-pl/110242

\title{
RESEARCH ON UNSTEADY CAVITATING FLOW AROUND A CLARK-Y $11.7 \%$ HYDROFOIL
}

\author{
Dorota Homa, Weodzimierz Wróblewski, Miroseaw Majkut, Michą Strozik \\ Silesian University of Technology, Institute of Power Engineering and Turbomachinery, Gliwice, Poland \\ e-mail: dorota.homa@polsl.pl
}

\begin{abstract}
The paper concerns experimental and numerical investigations focused on the cloud cavitation phenomenon over a hydrofoil. The results of flow visualization by means of a high-speed camera are presented. The cavitation cycles including vapour structures occurrence, development and collapse were recorded and described. Within the numerical investigation, transient calculations of cavitating flow were performed. OpenFOAM software was used. To model mass transfer between phases, the Kunz cavitation model was chosen. Turbulences were modelled by means of $k-\omega$ SST model. The vapour areas appearance, their shapes and changes in time were described and compared with experimental results. The characteristic features of cavitating flow were observed, however further adjustment of the cavitation model was advised.
\end{abstract}

Keywords: cavitation, flow simulation, flow visualization, homogeneous cavitation model

\section{Introduction}

Cavitation can be generally defined as a break of liquid flow continuity under very low pressure (Franc and Michel, 2004). It is a complex process that includes both vapour bubbles formation under low-pressure condition and their sudden collapses in the regions of higher pressure. These collapses are sources of pressure waves that propagate through the flow and can lead to walls or blades pitting. There are different types of flows with cavitation, but as far as turbomachinery performance is concerned, the main interest is focused on the flow over a hydrofoil. Cavitation in a pump or a water turbine can lead to many unwanted effects such as noise, vibrations, machine efficiency drop and erosion (Brennen, 1995), though it is strongly recommended to widen the knowledge about this highly unsteady phenomenon. Especially in the case of erosion risk assessment, it is necessary to identify locations of bubbles rebounds and collapses (Sedlár et al., 2012). The vapour bubbles can form structures of different shapes and dynamics of changes: transient isolated bubbles, partial cavities (attached, cloud cavitation and supercavitation) and tip vortex cavitation (Franc and Michel, 2004). The presented work is devoted to the partial cavities cloud cavitation regime and transition between cloud and supercavitation. The characteristic feature of cloud cavitation is the occurrence of the re-entrant jet, a jet of the liquid that forms at the phase interface near the wall at the foil suction side. It can be as thick as 0.15-0.3 maximum thickness of the cavitation structure (Callenaere et al., 2001) and travels upstream with the velocity magnitude close to the free stream velocity (Franc and Michel, 2004) penetrating the cavitation structure. The structure eventually detaches from the foil and collapses near the rear region of the blade. The re-entrant jet occurrence in the flow is the cause of cyclic changes of cloud cavitation. It was shown by Kawanami et al. (1997) that placing small obstacles on the wall can prevent the development of re-entrant jet and, as a consequence, the cloud cavitation does not form. 
The cavitation number $\sigma$ is defined as follows

$$
\sigma=\frac{p_{\infty}-p_{\text {sat }}}{0.5 \rho_{l} u_{\infty}^{2}}
$$

where: $p_{\infty}$ - free stream static pressure, $\mathrm{Pa} ; p_{\text {sat }}$ - vapour saturation pressure, $\mathrm{Pa} ; \rho_{l}$ - liquid density, $\mathrm{kg} / \mathrm{m}^{3} ; u_{\infty}$ - free stream velocity, $\mathrm{m} / \mathrm{s}$.

The lower cavitation number the larger cavitation structures can be observed in the liquid flow. The cavitation development is also influenced by the angle of attack of the blade as well as water quality (Arndt, 2012).

Experimental investigations of cavitating flow are usually performed in cavitation tunnels, in which conditions favourable to cavitation are evoked. Lowering the cavitation number can be reached by lowering the static pressure of the free stream flow or increasing the liquid velocity. The visualizations of cavitating flows are typically conducted by means of a high-speed camera in one view or two perpendicular views (Liu et al., 1999; Wang et al., 2001; Matsunari et al., 2012). Capturing high-frequency cyclic changes of the cavitation structures demands not only appropriate recording speed but also a relevant illumination system. To determine the velocity distribution in the cavitating flow, LDV or PIV techniques are used (Matsunari et al., 2012; Zhang et al., 1998).

Due to the rapid progress in computer science and computational fluid mechanics (CFD), simulating cavitating flow by means of CFD programs has become more and more popular recently. However, simulating cavitation in the flow can be a challenging task, due to the interfaces instabilities that result in explosion or collapse of cavities with large variations in size and velocity over short periods. The equation that describes the evolution of the bubble radius $R$ in time, known as the Rayleigh-Plesset equation states as follows

$$
\rho_{l}\left(R \ddot{R}+\frac{3}{2} \dot{R}^{2}\right)=p_{s}-p_{\infty}(t)-\frac{2 S}{R}-4 \mu \frac{\dot{R}}{R}
$$

where: $R$ - bubble radius, $\mathrm{m} ; S$ - surface tension coefficient, $\mathrm{N} / \mathrm{m} ; \mu$ - liquid dynamic viscosity, Pas. As the R-P equation is highly non-linear, some simplifications are introduced. The effects of surface tension and viscosity do not play a significant role in the bubble collapse process, comparing to the inertia forces (Franc and Michel, 2004). Equation (1.2) is then rewritten in the following form

$$
\frac{d R}{d t}=-\sqrt{\frac{2}{3} \frac{p_{\infty}-p_{s}}{\rho}}
$$

In the presented simulations, the homogeneous mixture model is applied. It is a commonly used approach to study cavitation flow numerically (Kunz et al., 2000; Schnerr and Sauer, 2001; Singhal et al., 2002; Zwart et al., 2004). The set of mixture conservation equations is solved (mass, momentum and energy), together with the continuity equation of the liquid or vapour phase. The continuity equation of the liquid phase can be written as

$$
\frac{\partial \rho_{l} \alpha_{l}}{\partial t}+\nabla \cdot\left(\rho_{l} \alpha_{l} u\right)=\Gamma
$$

and

$$
\Gamma=\left\{\begin{array}{lll}
\Gamma_{\text {cond }} & \text { if } & p>p_{s} \\
\Gamma_{\text {vap }} & \text { if } & p<p_{s}
\end{array}\right.
$$

where: $\alpha_{l}$ - liquid volume fraction, $-; t$ - time, $\mathrm{s} ; \rho_{l}$ - liquid density, $\mathrm{kg} / \mathrm{m}^{3} ; u$ - liquid velocity, $\mathrm{m} / \mathrm{s} ; \Gamma_{\text {cond }}, \Gamma_{\text {vap }}-$ mass transfer source terms (condensation and vaporization), $\mathrm{kg} /\left(\mathrm{m}^{3} \mathrm{~s}\right)$; 
$p$ - static pressure, Pa. The cavitation models differ from each other by the way the source term $\Gamma$ is determined.

In this study, the Kunz cavitation model is applied. The main aim of the study is to compare the results of cavitating flow visualization with corresponding simulation results to assess the applicability of a particular cavitation model. The cavitating flow visualization by means of a high-camera recording is presented. The authors included some results of this visualization in the case of incipient and developed cavitation in the previous paper (Homa et al., 2018). In this study, the results of cloud and transitional cloud to supercavitation regime are depicted in more a extended version (more frames per one cycle). Moreover, the comparison of obtained frequencies of changes in the whole range of investigated angles of attack and cavitation numbers is presented.

\section{Materials and methods}

\subsection{Experimental methods}

The investigated hydrofoil was installed on a rotary disc and placed in a rectangular chamber with three transparent walls made of acrylic glass (top, bottom and one side wall), which enabled flow observations. The Clark-Y profile was applied, with chord length $c$ equal to $70 \mathrm{~mm}$. The dimensions of measurement chamber were as follows: height equal to $189 \mathrm{~mm}(2.7 c)$, width equal to $70 \mathrm{~mm} \mathrm{(c)} \mathrm{and} \mathrm{length} \mathrm{equal} \mathrm{to} 700 \mathrm{~mm}(10 \mathrm{c})$. Change of the angle of attack was realized by the foil rotation over its aerodynamic center. It was located $210 \mathrm{~mm}(3 c)$ downstream the chamber inlet, in the middle of the chamber height. In Fig. 1 the outline of the test rig is depicted. Different levels of the cavitation number were obtained by changing the pump motor frequency.

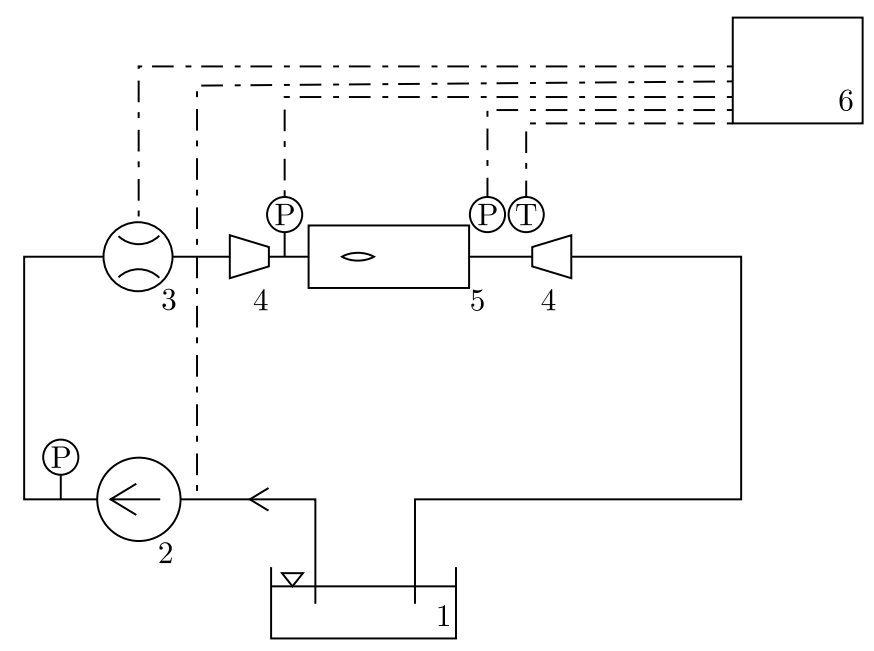

Fig. 1. The test rig. 1 - feeding water tank, 2 - pump, 3 - electromagnetic flowmeter, 4 - cross-section reduction, 5 - measurement chamber, 6 - computer unit, $P$ - pressure sensor, $T$ - temperature sensor

The water volumetric flow rate was increased as well as free stream velocity with a simultaneous decrease in the static outlet pressure. Regulation by frequency converter enabled smooth changes of flow parameters and variations of the cavitation number in the range between 2 to 0.7. The feeding tank of high capacity (over $35 \mathrm{~m}^{3}$ ) ensured almost constant water temperature during all measurements series. Pressure measurements were conducted by two piezoresistive pressure sensors (located at the inlet and at the outlet of the chamber) and temperature of the flow was measured by the resistance thermometer Pt100. All the measurement signals were collected by 
the computer unit. The relative uncertainty of the cavitation number determination was less than $1.3 \%$. The visualization of cavitating flow was performed by means of the high-speed camera Phantom Miro C110 with LED illuminator IL - 105/6X. The recording equipment at work is shown in Fig. 2.

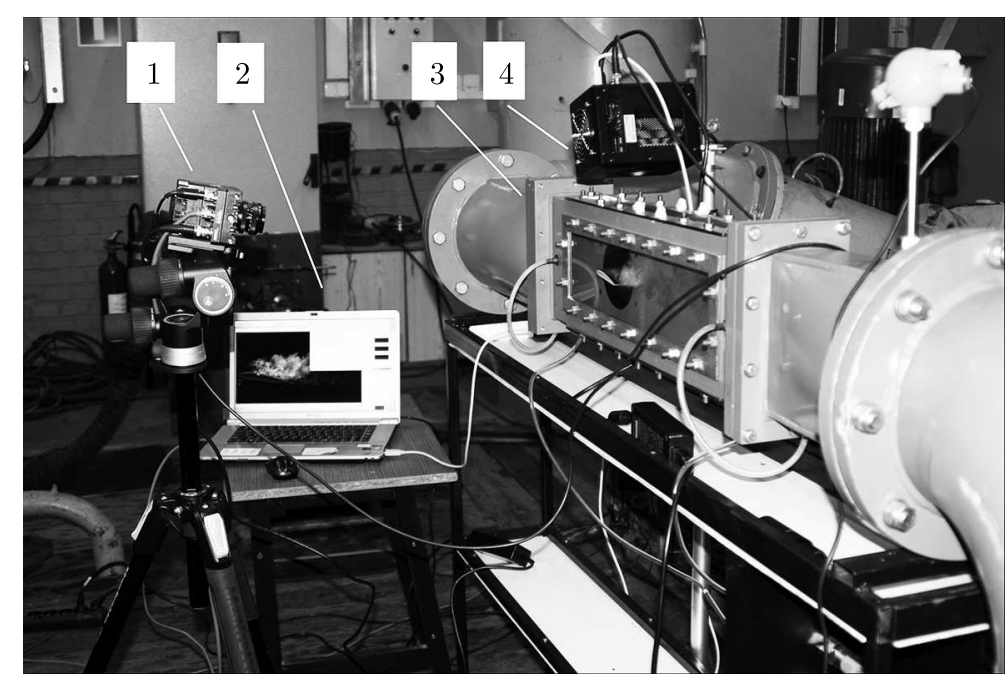

Fig. 2. Recording equipment. 1 - Phantom Miro C110 camera, 2 - computer unit, 3 - measurement chamber, 4 - illuminator IL-105/6X

Two camera parameters had to be set carefully when recording cavitating flow - recording speed and spatial resolution of the frames. During this investigation, the recording speed was equal to 1200 frames per second, which was high enough to capture the structures changes during one cavitation cycle, as well as sufficient spatial resolution was obtained $(1280 \times 720$ pixels). Next, the movies were split into a series of frames. On the basis of flow pictures, analysis of the frequency of changes for each case was carried out. Two different views were recorded - top and side view. As a result, both spanwise and streamwise distributions of cavitation structures could be described. One camera was used during the visualization, however the cyclic character of the recorded phenomenon enabled one to match top and side view pictures.

\subsection{Simulation setup}

Location of the foil in the computational domain was the same as during the experiment. The simulations were conducted in OpenFOAM software. Firstly, grid independence study was performed to determine the optimal nodes distributions. The grid was generated in ICEM CFD software. It was a $2 \mathrm{D}$ structural grid extruded to the overall width equal to $0.9 \mathrm{~mm}$ (3 layers of $0.3 \mathrm{~mm}$ thickness each). O-grid was generated around the blade, and the profile edge was divided into 4 parts: leading edge, upper side, lower side and trailing edge. Four different meshes were examined. Their parameters are summarized in Table 1.

Table 1. Parameters of grids tested during grid independence study

\begin{tabular}{|c|c|c|c|}
\hline $\begin{array}{c}\text { Mesh } \\
\text { symbol }\end{array}$ & $\begin{array}{c}\text { Number of } \\
\text { elements }\end{array}$ & $\begin{array}{c}\text { Number of nodes } \\
\text { around the foil }\end{array}$ & $\begin{array}{c}\text { Number of nodes in O-grid } \\
\text { (direction normal to the foil) }\end{array}$ \\
\hline \hline S1 & 93900 & 190 & 50 \\
\hline S2 & 116200 & 195 & 75 \\
\hline S3 & 155000 & 260 & 60 \\
\hline S4 & 160000 & 270 & 81 \\
\hline
\end{tabular}


Grid independence study included calculations with a cavitation number high enough to observe only one phase flow. The pressure distribution along the foil was compared with experimental results obtained by Matsunari et al. (2012) for the same foil. Pressure coefficient $C_{p}$ is determined as follows

$$
C_{p}(x)=\frac{p(x)-p_{\infty}}{0.5 \rho u_{\infty}^{2}}
$$

The pressure coefficient distribution along the foil is depicted in Fig. 3. The mesh with symbol S4 gave best results compared to the experiment, thus it was chosen for further computations.

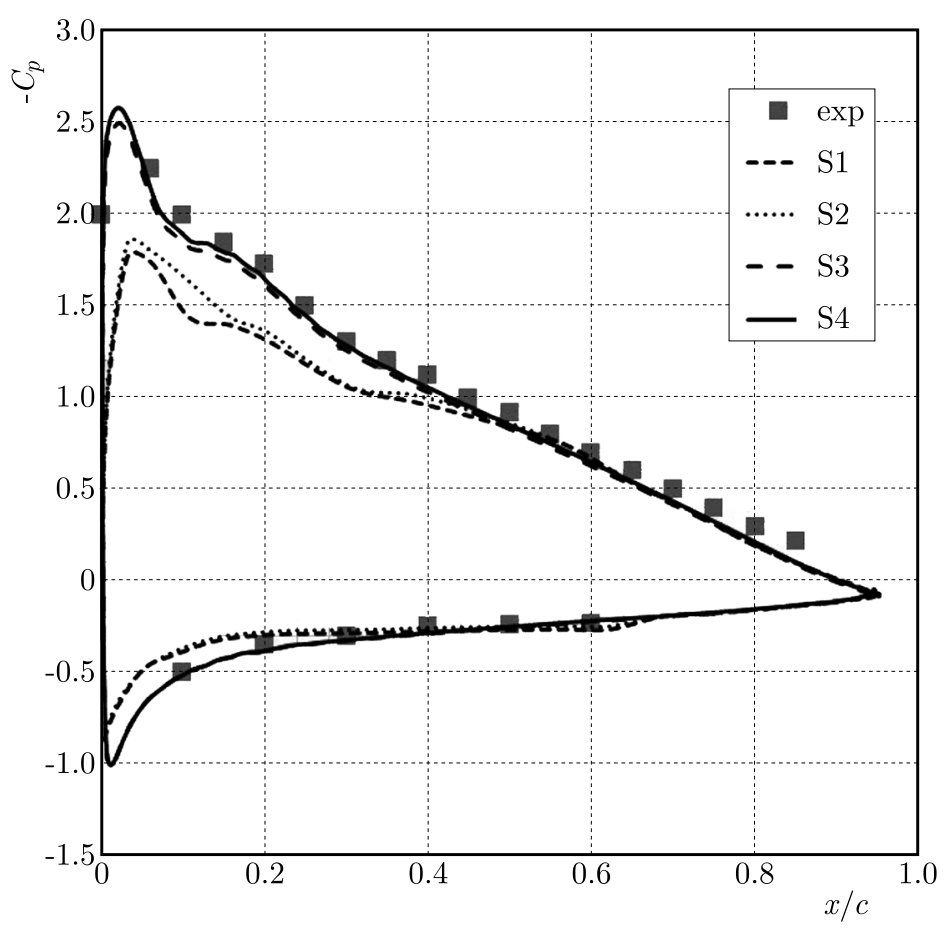

Fig. 3. Pressure coefficient distributions for different grids

The Kunz homogeneous cavitation model (Kunz et al., 2000) was used in the numerical simulations. To calculate the liquid phase, mass fractions equation (1.4) is solved, with the following source terms

$$
\Gamma_{\text {cond }}=\frac{C_{c} \rho_{v} \alpha_{l}^{2}\left(1-\alpha_{l}\right)}{t_{\infty}} \quad \Gamma_{\text {vap }}=-\frac{C_{v} \rho_{v} \alpha_{l}\left(p_{\text {sat }}-p\right)}{0.5 \rho_{l} u_{\infty}^{2} t_{\infty}}
$$

where: $\rho_{v}$ - vapour density, $\mathrm{kg} / \mathrm{m}^{3} ; u_{\infty}$ - free stream velocity, $\mathrm{m} / \mathrm{s} ; t_{\infty}-$ mean flow timescale, s; $C_{c}, C_{e}$-empirical coefficients dependent on the type of the flow.

Inlet velocity and outlet static pressure boundary conditions were applied. The top and bottom walls were assumed to be slip walls. The symmetry boundary condition was applied on the side planes of the computational domain. The simulations were conducted with interPhaseChangeFoam solver. This solver allows the phase change between two incompressible immiscible fluids on the assumption that the modelled flow is isothermal. Cavitating flow calculations have been conducted in OpenFOAM successfully for different foil types. Capurso et al. (2017) performed simulation of cavitation on Clark-Y foil, Hidalgo et al. (2014) used OpenFOAM to simulate unsteady cloud cavitation on NACA66 hydrofoil. Bensow and Bark (2010) conducted calculations in the case of NACA0015 hydrofoil with the LES turbulence model. In this study, the $k-\omega$ SST turbulence model was used. The turbulence intensity at the inlet was set to $5 \%$. Transient, 
the first-order Euler time scheme was used. The time step equal to $10^{-5} \mathrm{~s}$ was applied in the case of single phase calculations. For cavitating flow simulations the time step was reduced, set to the level that ensured the maximum Courant number value under 0.4. For the investigated range of the cavitation number time step varied from $10^{-6} \mathrm{~s}$ to $10^{-7} \mathrm{~s}$. The empirical coefficients $C_{e}$ and $C_{c}$ in the Kunz model were equal to 1000, according to the similar investigation conducted by Matsunari et al. (2012). During the calculations, the maximum value of $y+$ parameter on the foil wall reached 10, whereas the average value was equal to 2 .

\section{Results}

\subsection{Experiment results}

Four different levels of the cavitation number were recorded: $1.3 ; 1.0 ; 0.85$ and 0.75 with three different angles of attack $\alpha: 4^{\circ}, 6^{\circ}, 8^{\circ}$. Due to construction of the test rig, it was not possible to lower only one parameter in time (pressure or velocity). As it was an open tank installation, the change of flowrate caused the change of both flow velocity and pressure. The valve at the discharge line had to be fully open during the measurements to minimalize the pressure losses in the installation and to keep the low value of the head of pressure generated by the pump. The obtained cavitation numbers with the same rotational speed of the pump are close to each other. In each case, the movie was recorded and then split into separate frames. On the basis of frames analysis, the frequencies of changes were estimated. In Table 2, a summary of the obtained frequencies is shown.

Table 2. Summary of the experimental results

\begin{tabular}{|c|c|c|c|c|}
\hline \multicolumn{5}{|c|}{$\alpha=4^{\circ}$} \\
\hline \hline$\sigma[-]$ & 1.288 & 1.053 & 0.837 & 0.715 \\
\hline$f[\mathrm{~Hz}]$ & 37.5 & 28.6 & 25.5 & 15.8 \\
\hline Type & I & I & II & III \\
\hline \multicolumn{5}{|c|}{$\alpha=6^{\circ}$} \\
\hline \hline$\sigma[-]$ & 1.341 & 1.073 & 0.855 & 0.750 \\
\hline$f[\mathrm{~Hz}]$ & 33.3 & 20.3 & 18.8 & 17.6 \\
\hline Type & I & II & II & III \\
\hline \multicolumn{5}{|c|}{$\alpha=8^{\circ}$} \\
\hline \hline$\sigma[-]$ & 1.374 & 1.094 & 0.896 & 0.750 \\
\hline$f[\mathrm{~Hz}]$ & 35.3 & 22.6 & 18.7 & 16.7 \\
\hline Type & I & II & III & III \\
\hline
\end{tabular}

With a decrease in the cavitation number, for each angle of attack, the estimated shedding frequency declined. That means the cavitation structures lasted longer in the flow. On the basis of both frequencies values and shapes of structures, the measurement points can be categorised into three different types. The first type can be characterized as partial cavitation. The structures did not reach the foil chord and their maximum thickness was smaller than the maximum thickness of the foil. The frequencies of changes varied from about $38 \mathrm{~Hz}$ to $28 \mathrm{~Hz}$ (Table 2). The second type can be described as cloud cavitation. In Fig. 4 , an example of this type is depicted $\left(\alpha=6^{\circ}\right.$, $\sigma=1.073)$. To better visualize the results, colours inversion was applied. The cavitation cloud rose to about $40 \%$ of the period, then it started to detach from the foil wall. At $t / T=0.6$, the break of the structure is clearly visible. The smaller structure is attached while the greater one collapses in the rear region. At $t / T=0.85$ the growth of a new structure can be observed. The frequency of changes decreased to about $20 \mathrm{~Hz}$. 

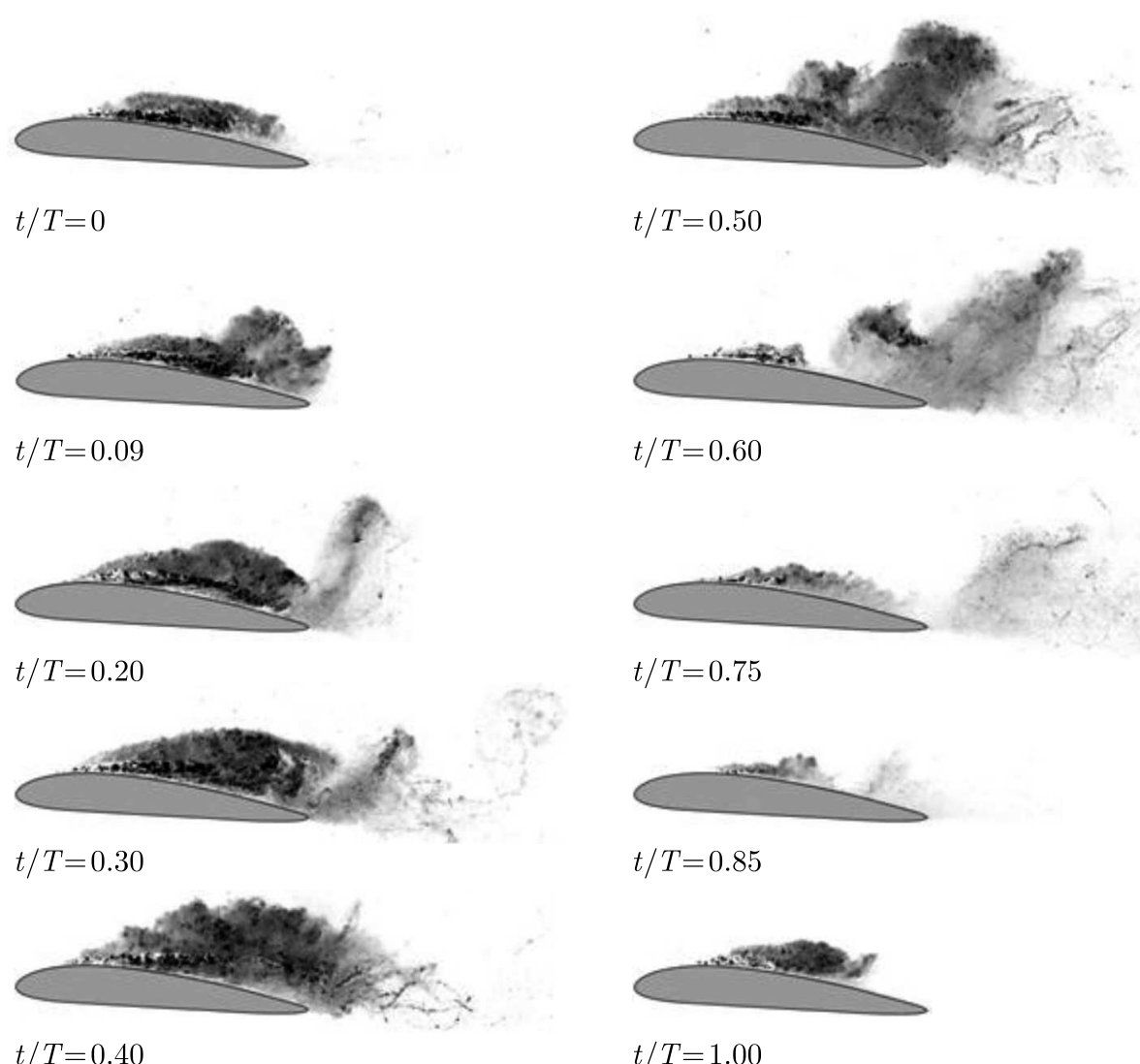

$t / T=0.50$

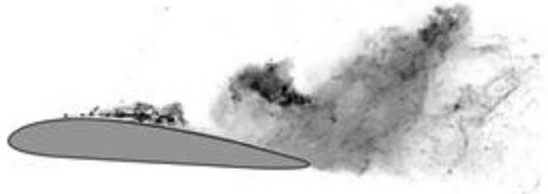

$t / T=0.60$

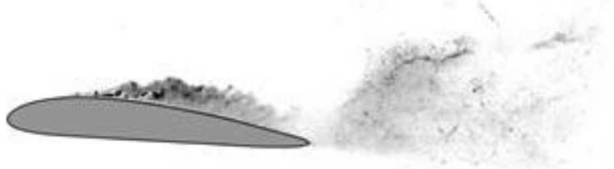

$t / T=0.75$

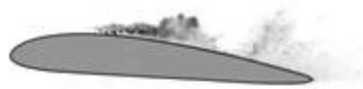

$t / T=0.85$

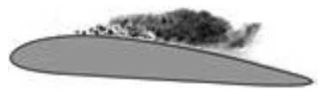

$t / T=1.00$

Fig. 4 . One cycle of cloud cavitation, $\alpha=6^{\circ}, \sigma=1.073, T=49.2 \mathrm{~ms}$, side view

The third type of observed cavitation can be described as a transition from cloud to supercavitation. Throughout most of the cycles, the length of the structure is greater than the foil chord length, which is assumed to be one of the criterions for supercavitation (Franc and Michel, 2004). However, the periodic changes of structures including both detachment and collapse were noticed, which indicated the cloud character of cavitation. In Fig. 5, one cycle of III type cavitation is shown. The structures became significantly longer and thicker compared to II type structures. In the top view, cavitation structures occupied the whole width of the foil almost all the time. The beginning of the structures moved slightly further from the leading edge comparing to the second type. The observation of top view pictures gives also information about bubbles distribution in the streamwise direction. Up to $20 \%$ of the foil chord the tiny, separated bubbles are observed. Going further downstream along the foil, the bubbles become bigger and form a cluster of different shapes. In the case of type II and III cavitation, the estimation of frequency values was quite challenging due to the fact that in these cases there were no clear moments of structure collapse and growth of a new structure. These phenomena happened concomitantly. The difficulty resulted from the illumination method used. All the structures in the spanwise direction were well lightened during visualization, which was an advantage in the case of determining the maximum outreach of the structure, but made it difficult to distinguish particular structures and assess their oscillations.

\subsection{CFD simulations results}

During the calculations, the vapour volume generated in the computational domain was monitored. On basis of the vapour volume fraction time course, the frequency of changes for each case was determined. In Fig. 6, the vapour volume fraction changes in time are depicted. 


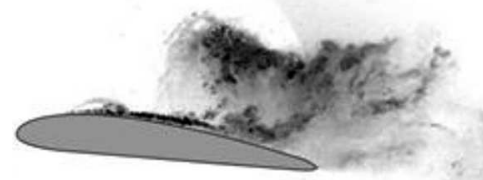

$t / T=0$

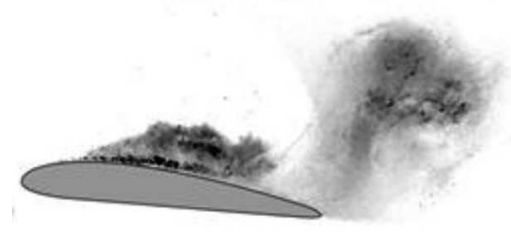

$t / T=0.10$

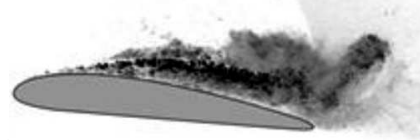

$t / T=0.20$

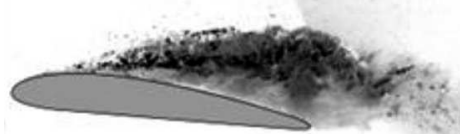

$t / T=0.33$

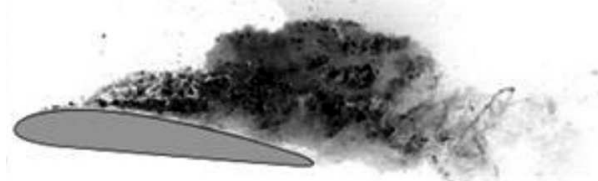

$t / T=0.45$

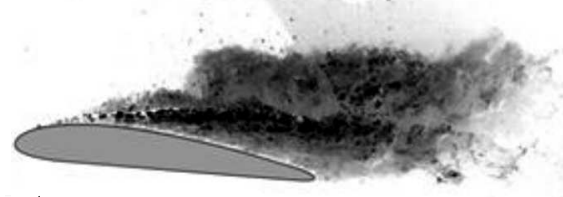

$t / T=0.55$

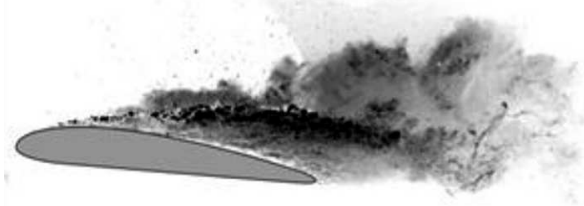

$t / T=0.65$
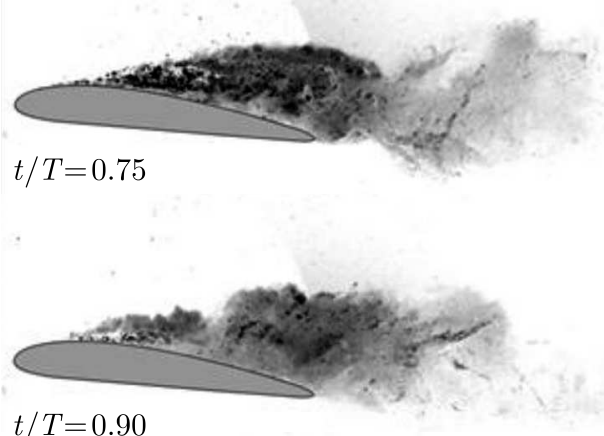

$t / T=0.90$

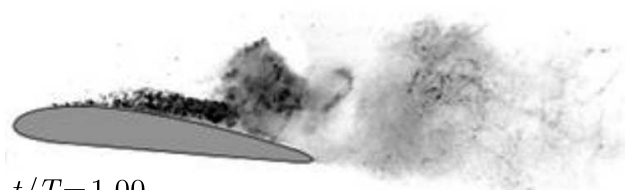

$t / T=1.00$

Fig. 5. One cycle of cloud/supercavitation, $\alpha=8^{\circ}, \sigma=0.750, T=60 \mathrm{~ms}$, side view

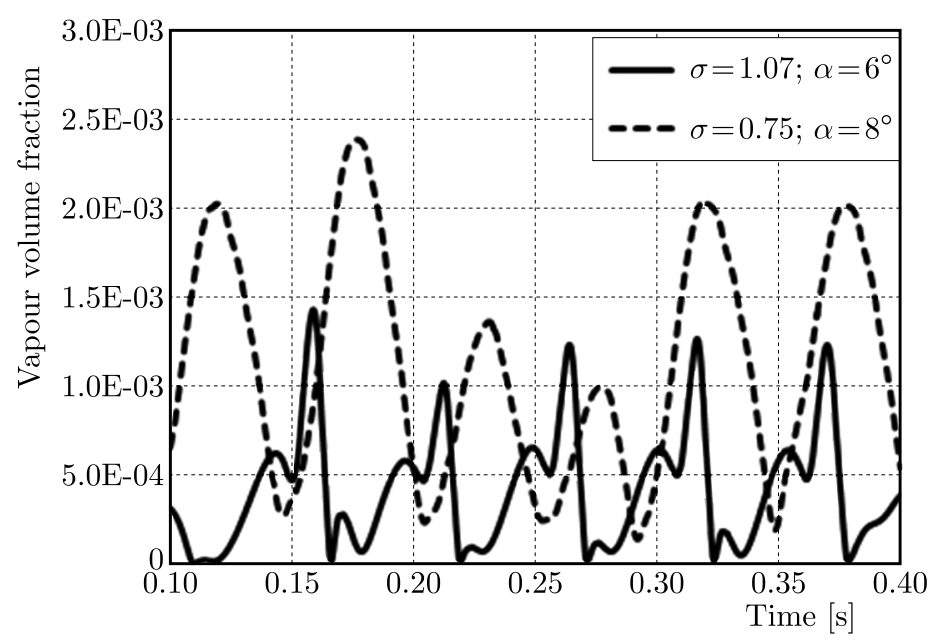

Fig. 6. Vapour volume fraction in the computational domain as a function of time 
For the cloud cavitation case $\left(\alpha=6^{\circ}, \sigma=1.073\right)$, the vapour volume generated in the domain periodically dropped to zero. For transitional cloud/supercavitation $\left(\alpha=8^{\circ}, \sigma=0.75\right)$ there were no moments without vapour in the flow, but the course of this parameter was more smooth. The average value of the vapour volume fraction was 2.4 times higher than in the case of cloud cavitation. The forces acting on the foil were observed too. After the calculations were completed, both lift and drag coefficients were determined. Their courses in time are presented in Fig. 7. For the cloud cavitation case both coefficients changed in a more irregular way than in the case of transitional cavitation. The average value of the lift coefficient decreased from 0.77 to 0.65 as the cavitation became more intense. Meanwhile, the average value of the drag coefficient increased from 0.07 to 0.12 . These values cannot be compared with the performed experiment, because there was no force measurement in the test rig. However, the observed trend - lift decrease and drag increase with the development of cloud structures was described in the literature (Watanabe et al., 2014). The irregularity of cloud cavitation influences other effects such as noise and erosion. Therefore, cloud cavitation is considered to be a cavitation type of the highest destructive potential (Wang et al., 2001).

\subsection{Comparison of experiment and CFD simulations}

The comparison between frequencies obtained in the experiment and during the simulation is shown in Table 3. For low angles of attack $\left(4^{\circ}\right.$ and $\left.6^{\circ}\right)$ and high cavitation number, the numerical model failed to predict properly the development of cavitation. For other cases, the frequencies from the computations are similar to the values estimated from the experiment, in three cases even very close to each other $\left(\alpha=4^{\circ}\right.$ and $\sigma=0.837 ; \alpha=6^{\circ}$ and $\sigma=1.073 ; \alpha=8^{\circ}$ and $\sigma=0.896)$. On the basis of frequencies analysis, it can be stated that the Kunz model with model constants $C_{e}$ and $C_{c}$ set to 1000 properly predicts the dynamics of developed cavitation. However, the change of the model constant should be considered in the case of partial or incipient cavitation. The period of cloud cavitation selected to visualization is depicted in Fig. 8, with corresponding images obtained during the experiment.

Table 3. Frequencies comparison between experimental and numerical results

\begin{tabular}{|c|c|c|c|c|}
\hline \multicolumn{5}{|c|}{$\alpha=4^{\circ}$} \\
\hline \hline$\sigma[-]$ & 1.288 & 1.053 & 0.837 & 0.715 \\
\hline$f_{\exp }[\mathrm{Hz}]$ & 37.5 & 28.6 & 25.5 & 15.8 \\
\hline$f_{C F D}[\mathrm{~Hz}]$ & no cavitation & stable structure & 25.6 & 33.5 \\
\hline \multicolumn{5}{|c|}{$\alpha=6^{\circ}$} \\
\hline \hline$\sigma[-]$ & 1.341 & 1.073 & 0.855 & 0.750 \\
\hline$f_{\exp }[\mathrm{Hz}]$ & 33.3 & 20.3 & 18.8 & 17.6 \\
\hline$f_{C F D}[\mathrm{~Hz}]$ & stable structure & 19.23 & 30.46 & 31.06 \\
\hline \multicolumn{5}{|c|}{$\alpha=8^{\circ}$} \\
\hline \hline$\sigma \sigma-]$ & 1.374 & 1.094 & 0.896 & 0.750 \\
\hline$f_{\exp }[\mathrm{Hz}]$ & 35.3 & 22.6 & 18.7 & 16.7 \\
\hline$f_{C F D}[\mathrm{~Hz}]$ & 19.3 & 19.4 & 19.7 & 19.5 \\
\hline
\end{tabular}

In the case of simulation, the break of the vapour structure was observed as in the experiment. The smaller structure stayed attached to the foil while the larger one disappeared near the rear region. The structures obtained in CFD analysis are significantly less developed than the structures from the experiment. The vortex structures obtained from simulations are two-dimensional and their shape is determined by the turbulence model.

In Fig. 9, a comparison between the experiment and CFD is shown in the case of the most intense cavitation that was investigated in this study. Once again, the structures obtained in 

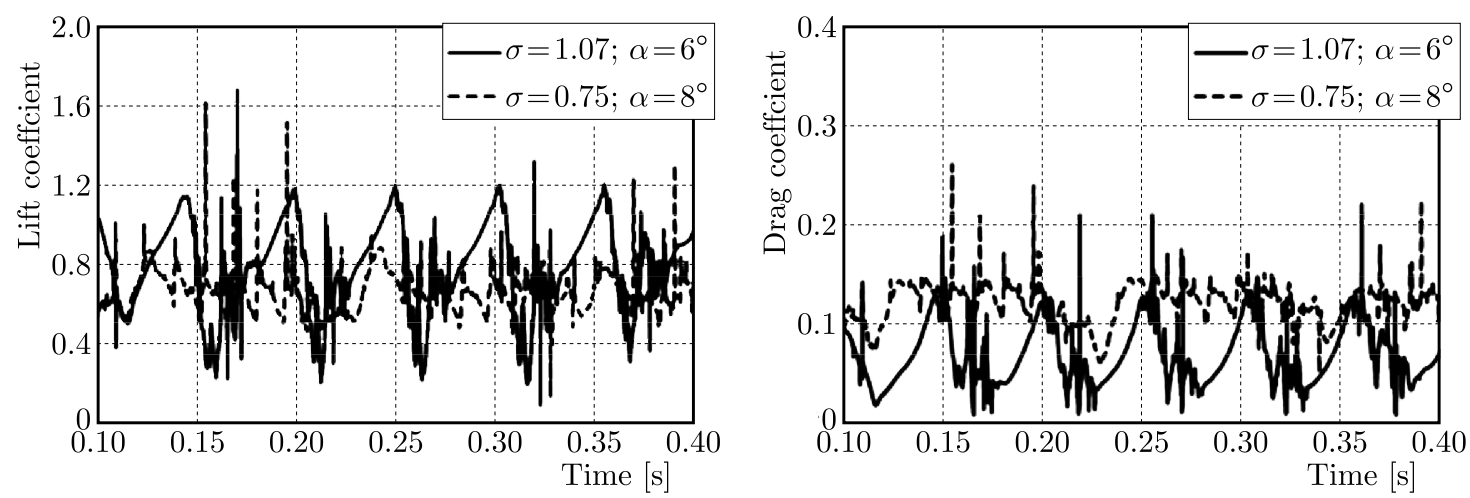

Fig. 7. Lift and drag coefficients in time

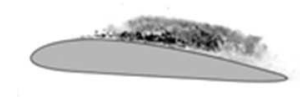

$t / T=0$

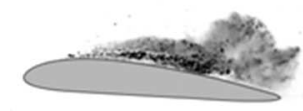

$t / T=0.09$

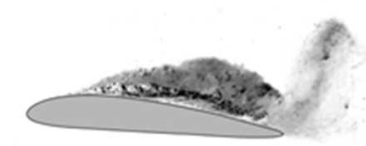

$t / T=0.20$

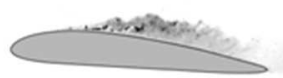

$t / T=0.75$

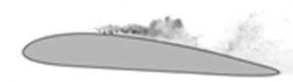

$t / T=0.85$
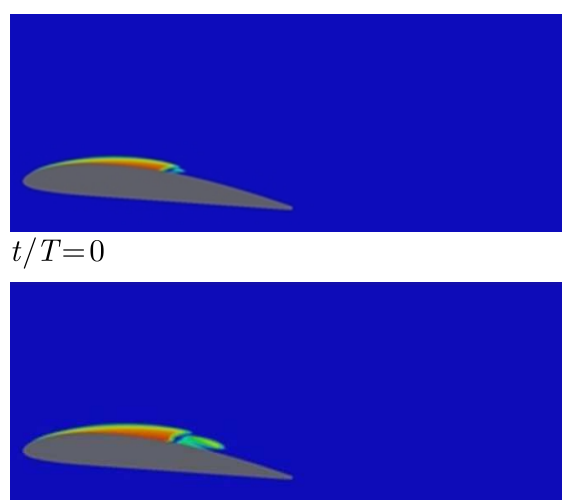

$t / T=0.11$

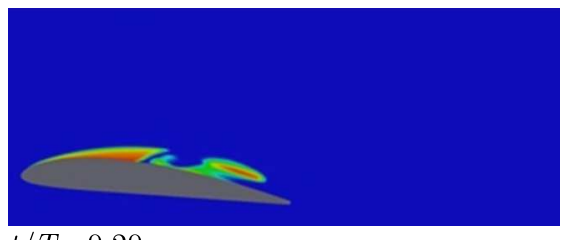

$t / T=0.20$

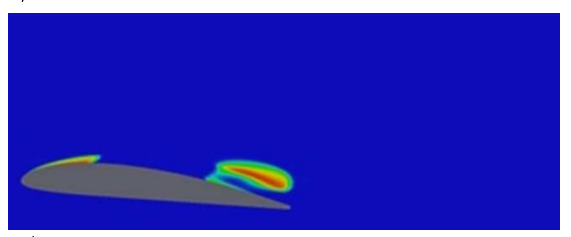

$$
t / T=0.75
$$

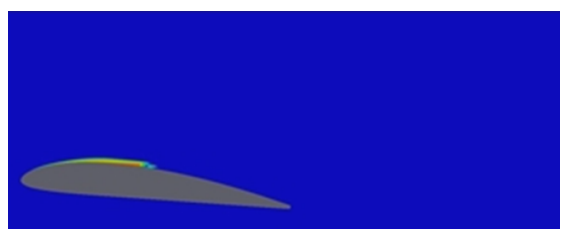

$t / T=0.85$

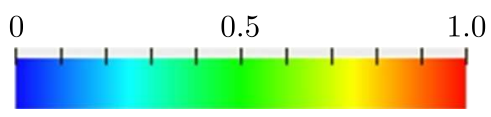

Vapour volume fraction $\alpha_{v}$

Fig. 8. Comparison of CFD and experiment, $\alpha=6^{\circ}, \sigma=1.073$; left - experiment, right - CFD 
simulations are smaller than the corresponding ones from the experiment. The mechanism of structure detachment was observed in the simulation. The vapour clouds form markedly more complicated structures than in the case of $\alpha=6^{\circ}, \sigma=1.073$ shown in Fig. 8. In $1 / 3$ of the period, even multiple splits of the structure can be observed - a tiny structure near the leading edge and a long structure attached to the foil, in which at least two clouds can be distinguished.

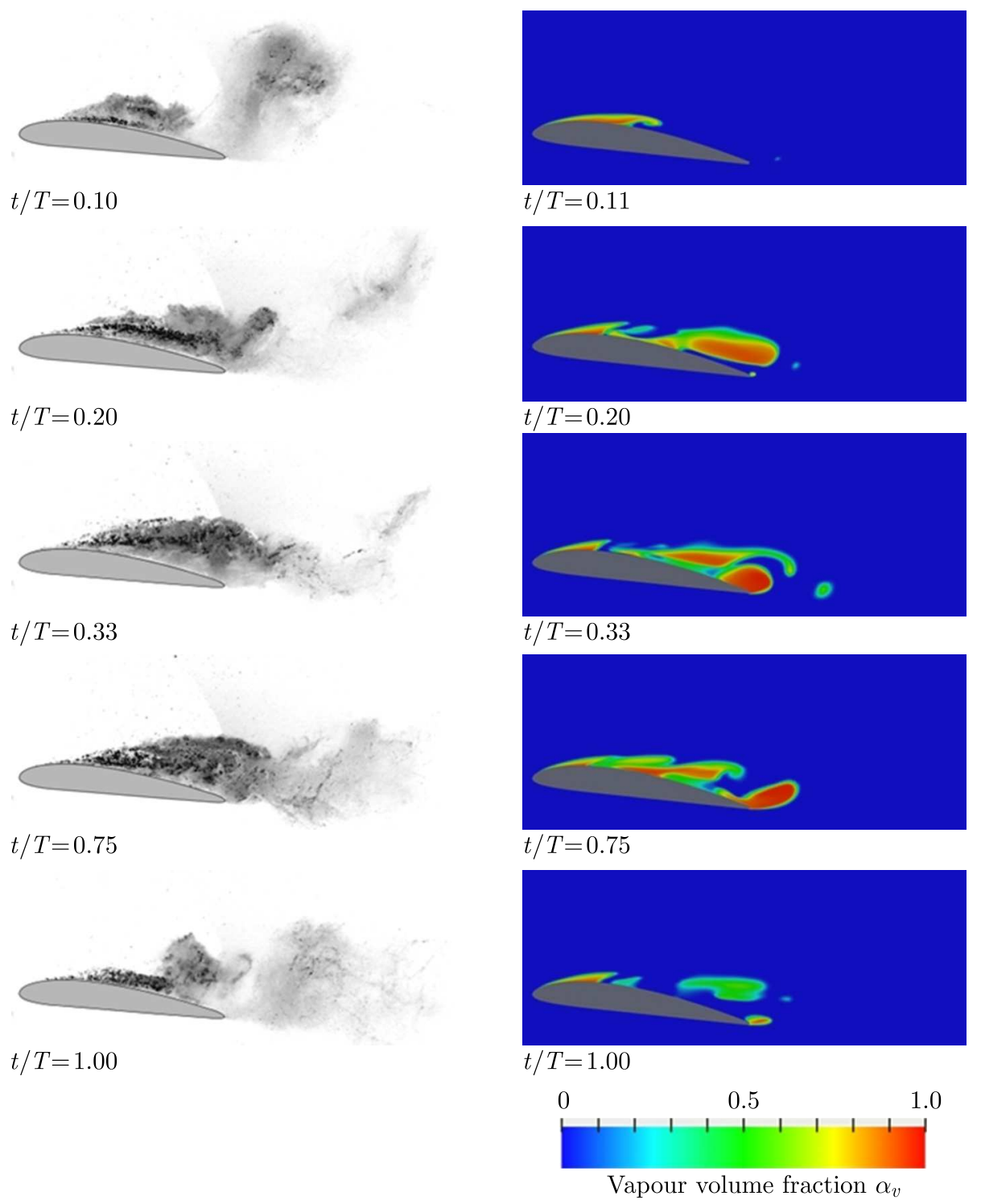

Fig. 9. Comparison of CFD and experiment, $\alpha=8^{\circ}, \sigma=0.750$; left - experiment, right - CFD

In the computational domain, the source of discrepancies between the size of the structures from the experiment and from CFD simulations is connected with a few issues. First of all, the simulations concerned 2D flow, thus what is observed in the pictures is only one layer of cavitating flow. In the case of the experiment in the frame, multiple layers in the spanwise direction are visible simultaneously, and it is not possible to extract only one from them. The maximum outreach of vapour structures is thus observed in these pictures. Secondly, the simulations were performed on the assumption of a two-phase flow. In reality, as the experiment was conducted in an open loop installation, there was some amount of air dissolved in the water, which was released under low-pressure conditions. The clouds observed in the experiment are in fact a mixture of vapour and air. Thirdly, the model coefficients $C_{e}$ and $C_{c}$ were set constant which enabled one to get stable results in the whole range of investigated angles of attack and cavitation 
numbers, but they might have blocked cavitation development under some conditions. They can be adjusted further for particular cases to get more similar images of the cavitating flow.

\section{Summary and conclusions}

In the paper, the results of both experimental and numerical investigations of cavitating flow over Clark-Y $11.7 \%$ foil are presented. The visualization of cavitation occurrence and development in the flow was performed by means of a high speed camera. After top view frames analysis, it was noticed that the structures exhibited non-uniformity in the spanwise direction. With a decrease in the cavitation number and increasing angle of attack, the vapour clouds occupied a larger part of the blade surface. The phenomenon of recurring growth, detachment from the foil wall and collapse of the structures near the blade rear region was observed and recorded. On the basis of image analysis, frequencies of changes were estimated. The numerical investigation included simulations of the flow under circumstances identical to the ones during the experiment. Although some simplifications were assumed, the obtained flow pattern was similar to the one observed at the test rig. The characteristic features of the cavitating flow were noticed. Moreover, during the calculations, the vapour volume fraction, drag and lift coefficients were monitored in the computational domain. These parameters cannot be compared with the performed experiment results, but the observed trends such as lift decrease and drag increase with the development of cavitation structures or cloud cavitation irregularity were also observed by other researchers. For most investigated cases, the obtained frequency of changes was close to experimental values. However, in a few cases, the numerical model failed to predict cavitation oscillations or even cavitation occurrence. The unsteady cavitating flow is strongly related to the well-developed turbulent structures. That leads to the conclusion that the selected cavitation model can be used to simulate this type of flow, but to improve the accuracy of predicted shedding frequency and outreach of vapour structures more advanced models are recommended.

Acknowledgements

The presented work was supported by the Polish National Science Centre founds within the project UMO-2016/21/B/ST8/01164.

\section{References}

1. Arndt R.E.A., 2012, Cavitation research from an intetrnational perspective, IOP Conference Series: Earth and Environmental Science, 15, Part 1, 227-238

2. BEnsow R.E., BARK G., 2010, Simulating cavitating flows with LES in OpenFOAM, 5th European Conference on Computational Fluid Dynamics, ECCOMAS CFD, 1-18

3. Brennen C., 1995, Cavitation and Bubble Dynamics, Cambridge University Press, New York

4. Callenaere M., Franc J.P., Michel J.M., Riondet M., 2001, The cavitation instability induced by the development of a re-entrant jet, Journal of Fluid Mechanics, 444, 223-256

5. Capurso T., Lopez M., Lorusso M., Torresi M., Pascazio G., Camporeale S.M., Fortunato B., 2017, Numerical investigation of cavitation on a NACA0015 hydrofoil by means of OpenFOAM, Energy Procedia, 126, 794-801

6. Franc J.P., Michel J.M., 2004, Fundamentals of Cavitation, Kluwer Academic Publishers, Dordrecht

7. Hidalgo V.H., Luo X.W., Escaler X., Ji J., Aguinaga A., 2014 Numerical investigation of unsteady cavitation around a NACA 66 hydrofoil using OpenFOAM, IOP Conference Series: Earth and Environmental Science, 22 
8. Homa D., Wroblewski W., Majkut M., Strozik M., 2018 Experimental and numerical investigation on Clark Y foil, IOP Conference Series: Journal of Physics, 1101

9. Kawanami Y., Kato H., Yamaguchi H., Tanimura M., Tagaya Y., 1997, Mechanism and control of cloud cavitation, Journal of Fluids Engineering, 119, 4, 788-794

10. Kunz R.F., Boger D.A., Stinebring D.R., Chyczewski T.S., Lindau J.W., Gibeling H.J., Venkateswaran S., Govindan T.R., 2000, A preconditioned Navier-Stokes method for two-phase flows with application to cavitation prediction, Computers and Fluids, 29, 8, 849-875

11. Liu S., Higuchi J., Ikohagi T., 1999, Experimental study of cavity flow behavior on a 2-D hydrofoil, JSME International Journal, 42, 4, 641-648

12. Matsunari H., Watanabe S., Konishi Y., Suefuji N., Furukawa A., 2012, Experimental/numerical study on cavitating flow around Clark Y 11.7\% hydrofoil, Proceedings of Eighth International Symposium on Cavitation, 358-363

13. Schnerr G.H., SAuer J., 2001, Physical and numerical modeling of unsteady cavitation dynamics, 4th International Conference on Multiphase Flows, New Orleans

14. Sedlář M., Komárek M., Wyroubal M., Müller M., 2012, Experimental and numerical analysis of cavitating flow around a hydrofoil, EPJ Web of Conferences, 25, 01084

15. Singhal A., Athavale M., Li H., Jiang Y., 2002, Mathematical basis and validation of the full cavitation model, Journal of Fluids Engineering, 124, 617-624

16. Wang G., Senocak I., Shyy W., Ikohagi T., Cao S., 2001, Dynamics of attached turbulent cavitating flows, Progress in Aerospace Sciences, 37, 6, 551-581

17. Watanabe S., Yamaoka W., Furukawa A., 2014, Unsteady lift and drag characteristics of cavitating Clark Y-11.7\% hydrofoil, IOP Conference Series: Earth and Environmental Science, 22

18. Zhang Y., Gopalan S., Katz J., 1998, On the flow structure and turbulence in the closure region of attached cavitation, Twenty-Second Symposium on Naval Hydrodynamics

19. Zwart P., Gerber G., Belamri T., 2004, A two-phase flow model for prediction cavitation dynamics, Proceedings of 5th International Conference on Multiphase Flow, Yokohama 